Results Statistical significance was assessed using the MannWhitney U test (for non-parametric data) and the Student's Ttest (for parametric data). Statistically significant differences between black and white HCM patients were noted in the $\%$ predicted peak power and $\%$ predicted peak $\mathrm{VO}_{2} / \mathrm{kg}$. (Table 1)

\begin{tabular}{|c|c|c|c|}
\hline & \multicolumn{2}{|c|}{ Mean values } & \multirow{3}{*}{$\begin{array}{c}\mathrm{p} \text {-value } \\
\text { (2-sided) }\end{array}$} \\
\hline & Black & White & \\
\hline & HCM & HCM & \\
\hline Age (years) & 45.08 & 45.47 & $0.9214^{*}$ \\
\hline BMI $\left(\mathrm{kg} / \mathrm{cm}^{2}\right)$ & 26.86 & 27.96 & $0.416^{*}$ \\
\hline Peak R achieved & 1.19 & 1.20 & $0.3576^{* *}$ \\
\hline$\%$ predicted $\mathrm{VO}_{2} / \mathrm{HR}$ & 95.77 & 102.42 & $0.177^{* *}$ \\
\hline $\mathrm{VE} / \mathrm{VCO}_{2}$ slope & 31.72 & 29.43 & $0.3524^{* *}$ \\
\hline \% predicted peak power (Watts) & 80.92 & 104.89 & $0.0016^{* *}$ \\
\hline Peak VO $/$ /kg (mls/min/kg) & 25.93 & 27.53 & $0.7114^{* *}$ \\
\hline$\%$ predicted peak $\mathrm{VO}_{2} / \mathrm{kg}(\mathrm{mls} / \mathrm{min} / \mathrm{kg})$ & 77.85 & 88.03 & $0.0434^{* *}$ \\
\hline $\begin{array}{l}\text { V02 at lactate threshold (\% of peak } \mathrm{V}_{2} / \\
\mathrm{kg} \text { ) }\end{array}$ & 58.88 & 55.66 & $0.3472^{* *}$ \\
\hline
\end{tabular}

Conclusion Black HCM patients achieve a significantly lower\% predicted peak power on CPET (24\% lower) compared with white HCM patients. Black HCM patients also demonstrate a significantly lower $\%$ predicted peak $\mathrm{VO}_{2} / \mathrm{kg}$ (10\% lower) compared to white HCM patients. Larger studies are required to corroborate these ethnic differences, however, this study suggests that the current standard cut-off of a peak $\mathrm{VO}_{2}>120 \%$ predicted may be too high for a black athlete resulting in a false positive diagnosis of HCM.

\section{INCIDENCE OF INFECTIVE ENDOCARDITIS IN UK: A MULTICENTRE RETROSPECTIVE ANALYSIS}

Joseph Stansfield*. Stepping Hill

\subsection{6/heartjnl-2017-311726.133}

Background Infective Endocarditis (IE) occurs in groups of people who are deemed high risk. Previously high-risk individuals undergoing invasive procedures were recommended prophylactic antibiotics. In 2008 NICE issued new guidance to avoid prophylactic antibiotics use.
Aim We aimed to evaluate the incidence of confirmed Infective Endocarditis in two sites across the North West region prior to and after the implementation of the 2008 guidance, including causative infective organisms, Echo findings, subsequent morbidity and mortality.

Methods OPCS-4 (Office of Population, Censuses and Surveys classification-4th Edition) system and the standard code allocated were retrospectively analysed for all admissions related to Infective Endocarditis for the years 2007-2008 and then again for the years 2012-2015.

Results The incidence of IE dramatically increased for the years after the guidance came in to effect compared to prior to the guidance. Initial data from one sites showed an increase in total numbers of cases year on year. All except 3 patients had positive blood cultures. Our data also shows that mortality remains high due to IE $(2012 \%-18 \%$ of patients died as a direct result of IE, 2013\%-38\%, 2014\%-11\%, 2015\%-21\%). Conclusions Incidence of IE has increased subsequently to the universal change in NICE guidance. Mortality associated with IE being still high. Although this is a small initial study from one centre, data from our second site is being collated to add support to our findings.

\section{SOURCES OF STREPTOCOCCAL BACTERAEMIA AND THEIR IMPLICATIONS FOR THE DIAGNOSIS OF INFECTIVE ENDOCARDITIS}

Louis Baig*, Mirza Wazir Baig, Jonathan Sandoe. Leeds Teaching Hospital Trusts

\subsection{6/heartinl-2017-311726.134}

Introduction The diagnosis of infective endocarditis (IE) is dependent on the detection of a sustained bacteraemia with multiple positive blood cultures being major criteria in the Duke nosology for IE. The interpretation of a single positive blood culture growing pathogens that could cause IE, but that do not fulfil major Duke criteria, is a common diagnostic difficulty in patients with a febrile illness. This study was designed to examine the clinical outcomes in patients with streptococcal bacteraemias and to determine the proportion of these with a final diagnosis of IE.

Methods This was a retrospective descriptive analysis of patients with streptococcal bacteraemias between SeptemberDecember 2012. IE was confirmed by a Consultant Microbiologist (JS) using the modified Duke criteria. The variables

Abstract 135 Table 1 Distribution of streptococcal species isolated in blood cultures and their aetiological role in cases of infective endocarditis according to age less than or greater than 18 years.

\begin{tabular}{|l|c|c|c|c|c|}
\hline & \multicolumn{2}{|c|}{ Age <18 } & \multicolumn{2}{c|}{ Age $\geq 18$} & Total \\
\hline & IE (\%) & Not IE (\%) & IE (\%) & Not IE (\%) & \\
\hline Streptococcus pneumoniae & $0(0)$ & $4(100)$ & $0(0)$ & $27(100)$ & 31 \\
\hline Oral streptococci & $0(0)$ & $16(100)$ & $5(12.5)$ & $35(87.5)$ & 56 \\
\hline Strep. bovis group (gallolyticus) & $0(0)$ & $0(0)$ & $0(0)$ & $1(100)$ & 1 \\
\hline Beta-haemolytic streptococci & $0(0)$ & $5(100 \%)$ & $2(12.5)$ & $14(87.5)$ & 21 \\
\hline $\begin{array}{l}\text { Strep. anginosus group } \\
\text { (anginosus/intermedius/constelatus) }\end{array}$ & $0(0)$ & $1(100 \%)$ & $0(0)$ & $2(100)$ & 3 \\
\hline
\end{tabular}


recorded were age, gender, number of blood cultures taken and the final diagnosis stated in the discharge summary.

Results 112 episodes were identified in 72 females and 40 males (mean age 40 years, range $<1-97$ ). The mean number of blood cultures was 2.57 (range1-12) and 85 (76\%) patients had only one blood culture taken. The infections recorded are shown in Figure 1. The cause of the bacteraemia was not stated in $33(29.5 \%)$ cases. Community acquired pneumonia $(n=31)$ was the commonest infection, followed by catheterrelated bloodstream infection $(n=8)$ and then IE and soft-tissue infection (both $\mathrm{n}=7$ ). Details of the streptococcal species are shown in Table 1 and the results have been divided using age less than or greater than 18 years. $50 \%$ of bacteraemias were caused by oral streptococci. IE was confirmed in only 7 $(1.2 \%)$.

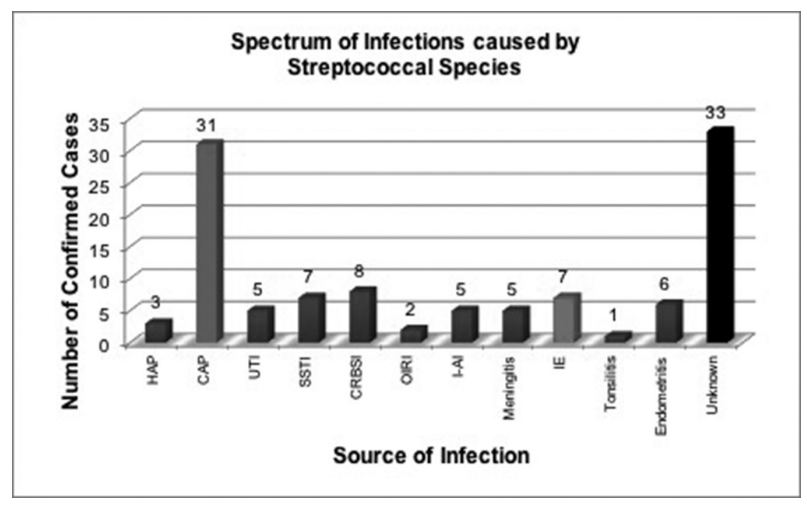

\section{Abstract 135 Figure 1}

Conclusions Among all streptococcal bacteraemias, IE is an uncommon cause but is more common when considering oral streptococci in adults (12.5\%) compared with those aged less than 18 years (0\%). Oral streptococci remain an important cause of IE particularly in adults. This diagnosis should be considered even if only one blood culture is taken and is positive. The high rate of single blood culture sampling (76\%) may be an unintended consequence of the Surviving Sepsis campaign and the drive to initiating empirical antibiotic therapy earlier. Under these circumstances the reliability of the Duke criteria will be reduced. Ideally, patients at increased risk of IE require multiple blood cultures if they have a significant pyrexial illness in order to determine if a sustained bacteraemia is present.

\section{6 GP AUSCULTATION FOR DIAGNOSING VALVULAR HEART DISEASE}

${ }^{1}$ Saul Myerson, ${ }^{2}$ Bernard Prendergast, ${ }^{1}$ Syed Gardezi ${ }^{*}$, ${ }^{1}$ Anthony Prothero, ${ }^{1}$ Andrew Kennedy, ${ }^{1}$ Joanna Wilson. ${ }^{1}$ Oxford University Hospitals NHS Foundation Trust; ${ }^{2}$ Guys and St Thomas Hospital NHS Foundation Trust

10.1136/heartjnl-2017-311726.135

Introduction Cardiac auscultation is an important clinical skill used by physicians in assessing and diagnosing valvular heart disease (VHD). The widespread use of echocardiography in the last three decades has coincided with a perceived decline in the utility of auscultation, particularly by general physicians. The ability of generalists to identify VHD in an unselected population has not been well characterised, so we aimed to determine the accuracy of auscultation in primary care for diagnosing VHD.

Methods 251 participants aged 65 and over who were participating in the OxValve population cohort study were included. They were recruited from two participating GP surgeries and had no previous diagnosis of VHD. The participants underwent cardiac auscultation during the OxValve study visit by two experienced General Practitioners (GPs), neither of whom had a specialist interest in cardiology. A 5-point Likert scale was used to rate the ability to hear heart sounds $(1=$ not at all; $5=$ perfectly) in addition to the presence or absence of a murmur, type of murmur and the ability to make a diagnosis based upon the auscultation findings. This was compared to transthoracic echocardiography performed at the same visit, but GPs were blind to the echocardiogram result, which was performed after auscultation. VHD was categorised as mild (either mild regurgitation [excluding trace/physiological] or aortic sclerosis) or significant (moderate/severe regurgitation or at least mild stenosis). Standard measures of diagnostic accuracy were calculated.

Results 82 murmurs were heard by the GPs (80 systolic; 2 diastolic). Echocardiography identified mild VHD in 174 $(69 \%)$ of the 251 participants, with more significant VHD present in $37(15 \%)$. The ability to hear a murmur on auscultation was not related to age, BMI or heart rate (table 1). Auscultation had a sensitivity of $32 \%$ and specificity of $67 \%$ for diagnosing mild VHD, which improved slightly for significant VHD to a sensitivity of $43 \%$, and specificity of $69 \%$ (table 2). The area under the curve on receiver operating characteristics (ROC) analysis was 0.50 for mild VHD and 0.56 for significant VHD (Figure-1) suggesting limited discriminatory ability.

Abstract 136 Table 1 The likelihood of hearing of murmur on auscultation and its relationship with age, BMI \& heart rate

\begin{tabular}{|c|c|c|c|}
\hline The likelihood of hearing of murmur on auscultation and its relationship with age, BMI \& heart rate \\
& $N$ & Correlation & Significance \\
( $p$ value)
\end{tabular}

